\title{
What is Interaction for Data Visualization?
}

\author{
Evanthia Dimara and Charles Perin*
}

\begin{abstract}
Interaction is fundamental to data visualization, but what "interaction" means in the context of visualization is ambiguous and confusing. We argue that this confusion is due to a lack of consensual definition. To tackle this problem, we start by synthesizing an inclusive view of interaction in the visualization community - including insights from information visualization, visual analytics and scientific visualization, as well as the input of both senior and junior visualization researchers. Once this view takes shape, we look at how interaction is defined in the field of human-computer interaction $(\mathrm{HCl})$. By extracting commonalities and differences between the views of interaction in visualization and in $\mathrm{HCl}$, we synthesize a definition of interaction for visualization. Our definition is meant to be a thinking tool and inspire novel and bolder interaction design practices. We hope that by better understanding what interaction in visualization is and what it can be, we will enrich the quality of interaction in visualization systems and empower those who use them.
\end{abstract}

Index Terms-interaction, visualization, data, definition, human-computer interaction.

\section{INTRODUCTION}

The notion of interaction has been a challenging concept to define in the field of Human-Computer Interaction (HCI). Only recently, an HCI review entitled "What is interaction?" [56] summarized concepts that describe the causal relationships between the human and the computer. While this HCI review outlines opportunities for enriching interactivity with computer systems, it is unclear how these concepts relate to visualization. Therefore, while visualization researchers and practitioners may be aware of such interaction concepts, they do not necessarily see how to apply them to their own data-oriented practices and needs.

Meanwhile in the past decade we have witnessed a growing call for enriching interactivity in visualization systems. Forward-looking research on interaction for visualization advocate for visualization systems that give absolute freedom to end users to actively restructure [73, 100], sketch [75], author [69,119] and personalize [57,111] visualizations; to construct visualizations from scratch [59], perform data-aware annotations on them [54,117], and unruly remove distracting information [29]; to enrich visualizations with external knowledge [118], control fluently [36] both data presentations [101] and data pre-processing statistical functions [37]; to indicate uncertainty [82], collaborate with peers $[55,80]$, and interact with visualizations using natural means $[66,74]$ within physically situated settings $[63,73,121]$.

We argue that a strong barrier to achieving this vision is not only that of the technical challenges, but like in HCI, that of defining interaction for visualization. In the visualization pipeline [16], interaction occurs at all stages of the visualization process of turning raw data into views on the data. While the visualization community has iteratively structured and formalized the representation aspect of the pipeline, significantly less attention has been paid to the interaction aspect [36, 72, 74, 99]. The nature and role of interaction has actually sparked discussions and arguments since the visualization field was created. As of today there is no consensus on what interaction is, and what its role for visualization is - as interaction is an elusive and overloaded term [74,92,126].

To address this problem, we first capture the current view of interaction from the visualization community based on the input of researchers. Once this view takes shape, we revisit the view of interaction from the HCI community [56] to understand how the two views differ and relate to each other. Combining these two perspectives, we then propose a

\footnotetext{
- Evanthia Dimara is with Sorbonne University. E-mail: evanthia.dimara@gmail.com.

- Charles Perin is with University of Victoria. E-mail: cperin@uvic.ca.
}

${ }^{*}$ All authors contributed equally to this manuscript, with the exception of the first author who did most of the work.

This is the author's version of the work.

To appear in IEEE Transactions on Visualization and Computer Graphics. definition of interaction for visualization. This definition attempts to broaden the scope of interaction in visualization and is inclusive as it considers the perspectives of information visualization, visual analytics, and scientific visualization. We further extend this definition to operationalize flexibility within visualization systems, based on where interactions occur semantically in the visualization pipeline. We hope that this definition will spur novel, bolder interaction design practices in visualization, and the growth of more flexible visualization tools.

\section{A ReView of Interaction for Visualization}

To capture the view of interaction in visualization, we started with a list of papers based on our own expertise and expert input. Then we applied a recursive process to expand our review while accounting for our own bias. We describe our methodology rationale, method for collecting papers, questionnaire we sent to experts, paper collection method, and tagging method. Then we present the summary statistics of the review.

\subsection{Methodology Rationale}

The topic of interaction in visualization is broad; arguably most visualization papers mention interaction at a point. Thus we discarded the systematic review and instead opted for a critical review, that needs not be exhaustive but requires a more detailed examination of the literature [46]. Because critical reviews seek to identify the most significant items in the field [46], we set the three following requirements:

R1:: The view of interaction of the community cannot be captured only by citation number, it needs to include expert input.

R2:: The snowballing approach alone (starting with a set of seed papers and expanding using back- and forward-references) is not sufficient because it is biased by the selection of seed papers.

R3:: The review must not include HCI papers unrelated to visualization.

We used two metrics to measure the impact and relevance of a paper $P$ based on publication year $P_{y}$. Impact I $_{P}=\left(P_{c} / 10\right) /\left(\right.$ current $_{-}$year $\left.P_{y}+1\right)$ measures the importance of the paper to the community based on its number of citations $P_{c}$. Relevance $P_{P}=P_{f} /$ (current_year $-P_{y}+$ $1)+\left(P_{b}+1\right) /\left(P_{y}-1980+1\right)$ measures the relevance to the topic of interaction based on its number of forward references $P_{f}$ and backward references $P_{b}$ (i.e., papers that cite, and that are cited by, $P$ ) that contain both "interaction" and "visualization" in their title. 1980 is the year of the oldest paper [17] we collected. These metrics identify both highly relevant and impactful papers, while not relying solely on bibliometrics.

We set the inclusion criteria to Impact $_{P}>0.5$, keeping papers with roughly more than 10 citations a year, and to Relevance $P>0.2$, keeping papers with roughly more than two forward or backward references with the keywords "interaction" and "visualization" in their title every 10 year. These cutoffs (which are broad to prevent false negatives) include important papers but exclude some clearly non-relevant ones. 


\subsection{Review Questionnaire}

We sent visualization researchers an online form asking for: (1) definition papers, the papers that attempt to define interaction in visualization; (2) relevant papers to the topic of interaction; (3) their years of visualization experience; and (4) their interaction experience on a 7-point scale describing how often their own papers focus on interaction. There were also three optional fields: name, email, and comments.

\subsection{Method for Collecting Papers}

We created a list of seed papers with the following snowballing method:

Step 1: We started from our own list of 5 definition papers (R1).

Step 2: We sent the questionnaire to expert visualization researchers who suggested both definition and linked papers $(\mathbf{R} 1, \mathbf{R 2})$.

Step 3: We included in the list of seed papers each paper $P$ that:

- was included in our initial list of 5 definition papers or was suggested as a definition paper at least once; and

- is a journal article or conference paper - to avoid non-peer reviewed entries such as demos; and

- has the term "visualization" in the title or abstract (R3); and

- has Relevance $P>0.2$ and Impact $_{P}>0.5$.

In addition, considering that expert input can provide insights not captured by our computational method (R1), we included in the list of seed papers those that did not fulfill these inclusion criteria but that were suggested as definition paper three times or more.

Step 4: We repeated Step 2, sending the questionnaire to all (nonpreviously contacted) authors of the added seed papers. We then repeated Step 3 with the new suggestions. We stopped the recursion when no new seed paper or author was found.

Once the list of seed papers finalized, we derived a set of linked papers. A paper $P$ is linked if it is not seed and one of the following is true:

- $P$ is suggested by two or more experts as definition or relevant;

- $P$ cites or is cited by a seed paper and its title contains both "visualization" and "interaction".

\subsection{Method for Tagging Papers with Interaction Themes}

We (the two authors of this paper) developed tags (short labels) to characterize the seed and linked papers using an iterative deductive coding method [88]. Specifically, we identified emerging themes from reading seed papers with the lens of capturing: i) the current view of interaction in visualization; ii) problems raised by the community; and iii) existing archetypal descriptions and categorizations of interactions. We then refined these tags incrementally until we obtained a high inter-coder agreement. We then coded all seed and linked papers. We measured inter-coder agreement using Cohen's Kappa [24] $(K)$, that accounts for the possibility that agreement occurs by chance. There is no strict rule regarding how to interpret values of $K$, but inter-reliability is considered satisfactory for $K \geq 0.7$ and excellent for $K>0.75$ [41]

For the first coding iteration, we used plain tag names. We independently tagged 6 seed papers using the tag names described below with binary values (a paper either has the tag or not). We obtained $K=0.57$. We discussed similarities and differences in our tagging, then wrote the detailed definitions for each tag presented below. We switched to three values: 0 (not applicable tag), 1 (partial fit of the tag), and 2 (good fit of the tag). For the second iteration, we independently coded again the 6 first seed papers $(K=0.83)$, as well as 6 additional seed papers $(K=0.78)$, showing excellent agreement $(K>0.75)$. For the third iteration, we independently coded 12 new seed papers. We obtained $K=0.87$, which allowed us to have a single coder per remaining paper.

Tag - Definition of Interaction: attempt to define, explain or describe interaction. A rating of 1 marks implicit attempts, including definitions based on specific properties or attributes of interaction. A rating of 2 marks direct definitions (formal or informal, e.g., "interaction is ...").

Tag - Critical on Interaction: complaints and frustrations (using a negative tone) about interaction, to reveal areas of improvement and challenges. A rating of 1 marks brief complaints. A rating of 2 marks extensive discussions of complaints.
Tag - Benefits of Interaction: positive aspects of interaction (actual or expected). A rating of 1 marks brief (or moderately phrased) discussions of benefits. A rating of 2 marks more elaborated (or strongly phrased) discussions of benefits.

Tag - Interaction Concepts: archetypal description of interaction. Given to papers that contribute one of: "concept", "model", "framework", "design space", "paradigm". A rating of 1 marks concepts with small or unclear relation to interaction. A rating of 2 marks concepts with interaction as a key element (e.g., an interaction model).

Tag - Interactive Pipeline: discussion of interaction in relation to the visualization pipeline, a fundamental concept that transcends subdomains of visualization and explicitly includes interaction. It is given to papers that use the word "pipeline" or cite one of [16,20,21,48]. A rating of 1 indicates that the existence of interaction is unclear. A rating of 2 indicates that the interaction component is clearly discussed.

Tag - List: contribution of a list (e.g., of interaction techniques, tasks, or intents). We call formal a list that is described with one of the following words: "taxonomy", "classification", "typology", "categorization". A rating of 1 marks an informal review (it does not contain any of the formal keywords). A rating of 2 marks a formal review.

\subsection{Summary Statistics of the Review ${ }^{1}$}

RECRUITING: We emailed 77 visualization experts over a two-month period. 64/77 were successfully delivered. 34/77 were in our initial list of experts and the remaining 43/77 were derived through our recursive algorithm. While we started with a bias toward information visualization (e.g., $[36,72,79,106])$, our algorithm expanded the scope naturally to visual analytics (e.g., $[37,45,50,92])$, scientific visualization (e.g., [1,66, 67,77]) and digital cartography (e.g., [98, 99]).

PARTICIPANTS: 22 visualization researchers responded to the survey. They had 5-32 ( $M: 13.6, S D: 6.2)$ years of visualization experience. $12 / 22$ were authors of seed papers, and 14/22 were authors of linked papers; 2/22 completed the survey anonymously. Participants rated their interaction expertise with a mean of 5.9/7 ( $S D: 0.8)$.

SEED PAPERS: Fig. 1 presents all 59 tagged papers. We started with 5 definition paper based on our expertise. Participants suggested 114 (83 unique) definition papers (6 did not suggest any definition paper). Applying our recursive algorithm resulted in a set of 23 seed papers.

LINKED PAPERS: Participants suggested 61 (51 unique) relevant papers. Forward and backward searches gave 105 papers citing, and 104 papers being cited by a seed paper. Our linked paper collection algorithm gave 47 linked papers. We excluded 7 theses, 3 unpublished and 1 non-English reports. This resulted in 36 linked papers.

TAGS: The derived tags were: definition: 32; benefit: 39; critical: 36; concept: 20; pipeline: 12; and list: 35 .

\section{The Visualization View of Interaction}

In this section, we synthesize the current view of interaction in visualization based only on the points of view of the 59 seed and linked papers we reviewed (see Fig. 1). We elaborate on our own critique of this literature in Sect. 4 and Sect. 5. While we reviewed all 59 papers, due to space limitations, we only cite papers which have been cited more than twice - and provide all papers in supplementary material.

\subsection{Definitions of Interaction for Visualization}

Interaction has been identified as an ovloaded [92] and elusive [36,74] term, and it is challenging to find a solid definition of interaction $[36,74,126]$. Therefore, we consider the 32 papers with a definition tag - whether they attempt to define interaction implicitly or explicitly.

\subsubsection{What Interaction for Visualization Must Involve}

The definition papers reveal mandatory components of interaction, namely: external and internal entities, external and internal actions.

EXTERNAL ENTITIES: The two most cited mandatory entities are the user and the data. The user (or analyst $[8,37,112]$ ) is a human

\footnotetext{
${ }^{1}$ Material: https://osf.io/ej7xg/?view_only=51485163dfc94d0c8499af17cb2038b2
} 


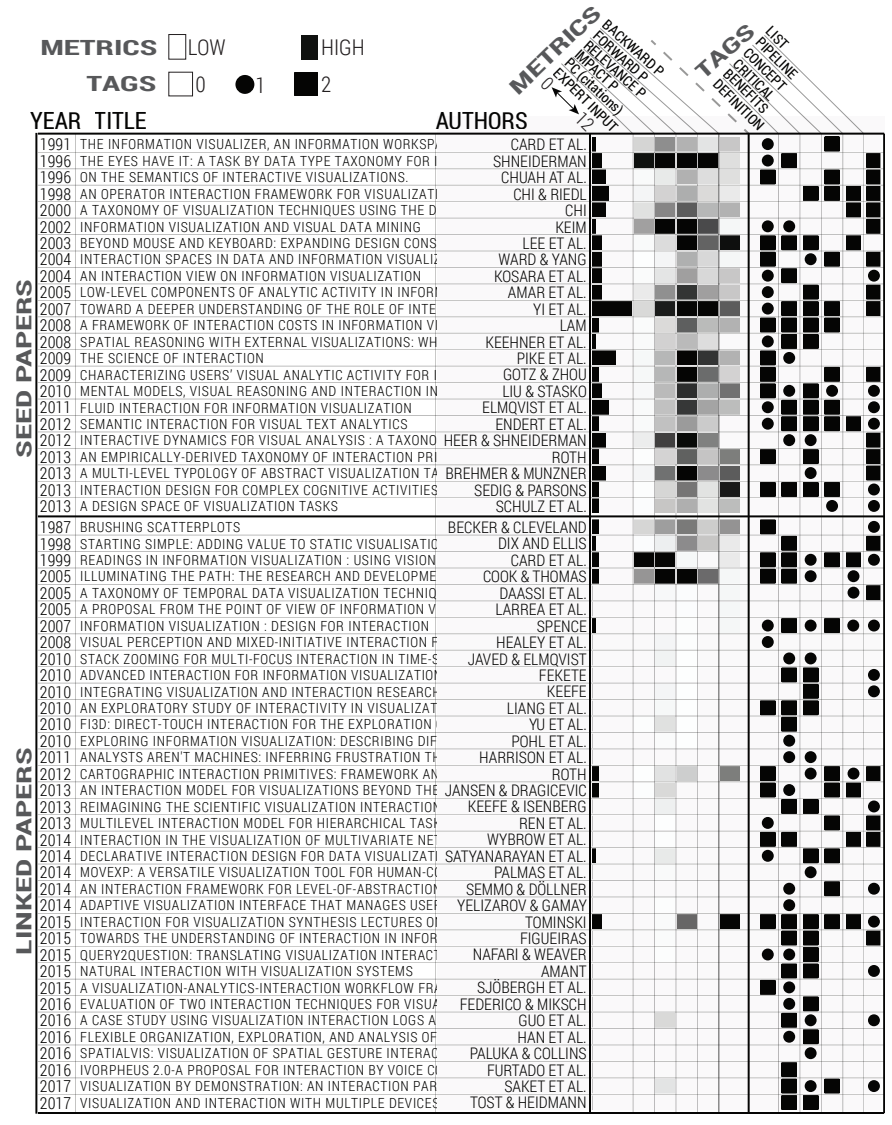

Fig. 1. The 23 seed and 36 linked papers in our review, ordered by year. It shows expert input, the algorithm metrics, and our tagging scores.

being who can be characterized by skills [36], abilities, expertise and motivation [99], and who initiates the interaction [104]. Some papers differentiate between end-users and designers [22]. The data is an intangible information source that is the user's main object of interest.

The mean with which the user interacts with the data is a mediating $[98,99]$ entity that we call the visualization system. The visualization system (either as a whole or some of its components) is referred to as technology [74, 99, 104], computational tools [92] and computer [8] with hardware and software [8], when emphasizing its technological properties; representation [76, 104,126], visualization $[16,19,22,45,67$, 68,72,79,99,120], map [98], graphical marks [2,22], display [8,74,106], visual metaphor [37] and non-static image [70], when emphasizing its visual properties; and interface [19,22,36,76,92,98,99, 104], system [19, 36, 45, 62, 74, 123, 126], analysis tool [92], workspace [19] and interaction space [104], when referring to a more abstract mediating entity. Some papers add that the mediating entity should provide a set of controls [92] for the user to access the data.

Most papers identify time as a fundamental entity of interaction [16, $62,76,126]$. Interaction, unlike representation [126], is seen as a realtime $[8,98]$ action with a start and an end $[74]$ that should result in an immediate response from the visualization system [16, 19,36, 106, 123]

INTERNAL ENTITIES: Interaction is characterized as a goal-oriented activity $[53,62,92]$ involving a data-oriented intent. Intent $[36,45$, $74,76,79,84,92]$ is also phrased as goal [45, 62, 76, 92, 97-99, 123], task $[45,67,106]$ or problem $[53,92]$. Intent can describe a high-level data exploration $[19,45,62,68,84,92,126]$, the generation of insights $[45,62,92,98,99,126]$, and the need to acquire multiple perspectives on the data $[62,92,104,126]$. Intent can go beyond exploration, such as the intent to collect and correct data [62], or social intents to coordinate in collaborative setups and to present data to an audience [62]. Intent is both identified at a low, operational level, e.g., to alter the representation $[19,126]$, and at a higher level, e.g., for information foraging [79], sensemaking [99, 104], and knowledge creation [92].
EXTERNAL ACTIONS: Interaction is described as a dialogue between the user and the visualization system $[36,76,92,98,104,123,126]$ made of action-reaction pairs [76, 104]. The user performs an action (or input $[22,102])$ on the visualization system $[45,67,72,76,79,104]$. The visualization system returns a reaction $[74,76,104]$ (or response $[19,67$, $74,76,98,106,120,126]$, change $[8,16,22,98,120,126]$, output $[22,102])$ that is perceived by the user $[72,104,120]$. This reaction has been called permutation of graphical marks [2], change of transformation parameters [123], and alteration of the pipeline [62].

INTERNAL ACTIONS: Along with its physical acts, interaction with a visualization system involves a cognitive act of the user $[37,92,104]$ or, similarly, a reasoning/analytic process $[67,76,79,97]$ on the data.

\subsubsection{What Interaction for Visualization Can Involve}

Interaction for visualization can involve additional external and internal entities, and additional external and internal actions.

EXTERNAL ENTITIES: Interaction can involve external physical objects $[62,74,106]$ such as mouse [79], pen [74] keyboard [79] and physical constraints [99]. It can also involve a variety of modalities such as body movements [19,74], speech [74], head [79] and eye movement [79]. Many modalities (e.g., gaze, head) tend to be overlooked in visualization. Restricting modalities leads to a "disjointed picture of human performance" [79] and lost opportunities to capture user intent [79]. Interaction also involves the environment under which interaction occurs, for example whether it is a casual or working environment [99], and whether there are multiple users involved [62, 74, 92, 99].

INTERNAL ENTITIES: Users' prior knowledge [92], internal mental representation [104], skills [36] and abilities [99] can be involved when interacting with a visualization system. While interaction can start with a concrete user query $[19,45,84,106]$, it can also lead to the internalization of new goals [104]. Moreover, interactions might occur with an absence of intent, such as with proxemics interactions [74].

EXTERNAL ACTIONS: Interaction can involve many user actions. Such action can change the data $[16,22,45,76,84,92,102,104]$, e.g., with filtering and aggregation [102]. It can change the representation and presentation of information [8,16, 19,37,45, 68, 79, 84, 104], e.g., with sorting [45] or when switching from a map to a timeline [45]. It can create metadata, for example by temporarily marking data to track, annotate or bookmark [37,45,84]. It can create new data representations $[74,79]$ and new data, to express and manipulate new knowledge [37, $45,104]$, e.g., with note taking and when manipulating a knowledge management component [45]. A user can perform an action that does not occur on the data or their representation, e.g. adjusting a movable baseline to compare the heights of a histogram [126], performing a metaction on their own action history, (undo/redo) [45], and steering a statistical data model [37]. Last, along with the dialogue between the user and the visualization system, there is also the dialogue of users with the external environment [92] and with their peers [62,74,92,99]. INTERNAL ACTIONS: While interaction with a data source is enabled within the context of a tangible visualization system, much of it can occur internally in user's mind [74,92], for example information processing [104], memory encoding [104], and simulative reasoning [79]. On a deeper level, the interaction takes the form of a dialogue between the "internal representations and processes of the user and the external representations and processes" of the visualization system [104].

\subsubsection{Properties of Interaction}

Interaction for visualization is characterized as a goal-oriented [62, $76,92,97-99,123]$ activity that contains semantics $[22,45]$, that is sequential [22, 45, 98, 104], incremental $[16,36,37,106]$, and iterative $[19,98,104]$, and preserves the following properties:

GRANULARITY: Interaction is characterized at multiple levels of granularity $[2,45,62,76,102,104]$. Such levels include micro-level (e.g., mouse clicks), macro-level (e.g. hypothesis generation) and levels in between (e.g., filter, sort). To distinguish these levels, terms such as \{subtasks, tasks, activities\} [2], \{events, actions, subtasks, tasks\} [45] and $\{$ events, streams, signals, predicates $\}$ [102] have been used. Yet, 
it is often unclear whether these levels refer to interaction per se or to user intent. The lack of distinction between levels of interaction results in no established conceptualization and vocabulary [104].

CONTINUOUS VS. DISCRETE: The temporal dimension, which is a fundamental entity of interaction (see Sect. 3.1.1), is either continuous or discrete [76,84]. Continuous interactions involve a sequence of intermediate visualization states between an initial state and a goal state (e.g., mouse drags to perform a lasso selection) [84]. Such interactions happen over a span of time [76]. With discrete interactions, action and reaction occur in a distinct manner [76] (e.g., clicking on a checkbox to toggle filtering [84]). Action and reaction can have different continuity (e.g., a continuous action can have a discrete reaction [76]).

DIRECT VS. INDIRECT: Directness is associated with continuous representation of the objects of interest, rapid, incremental, and reversible user actions; allowing for usage with minimal knowledge [16,36]. Directness was first associated with techniques such as dynamic queries [16] that provide widgets to explore the data interactively - as opposed to command-line interfaces. But the meaning of directness has changed and dynamic queries are now said to be indirect because the user in teracts with intermediate, likely spatially distant widgets - as opposed to interacting directly with the representation of the data itself. Rather than (quite arbitrarily) classifying interactions as either direct or indirect, it is useful to consider that interactions have degrees of directness on an indirect-direct continuum [62].

\subsubsection{Terms Related to Interaction}

Interaction is distinct from interaction technique, interactivity, and science of interaction. Interaction technique is less broad [104] and more tangible [126] than interaction, and refers to the user means with which interaction can occur [92] (in terms of hardware and software [62]). Unlike interaction, an interaction technique does not necessarily embed the notion of intent [92]. Whereas interaction refers to the action-reaction dialogue between a user and visualization system, interactivity refers to the feel, properties, and quality of this interaction [76]. Yet, the terms are sometimes used interchangeably $[21,62]$. Science of interaction is broader than interaction [92]. It is the study of methods by which humans create knowledge through interaction, and it involves developing and testing theories and practices to better support interaction [92]. All these terms internalize the notion of interaction. Thus, making them actionable requires a precise view of what interaction is.

\subsection{Reported Benefits \& Critiques on Interaction}

39 papers reveal benefits of interaction. At first, interaction was seen as a necessity to handle increasing amounts of data $[32,38,64,106,126]$ But interaction has moved beyond its necessity. It is now seen as a mean to amplify cognition in active, human-driven data exploration $[37,47,54,68,70,79,95,104,125,126]$ in which the user is in control of the information space $[36,100,106]$. It is via interactive manipulation that "knowledge is constructed, tested, refined and shared" [92]. Further, interaction leverages humans' natural abilities through new visualization shapes, modalities, and input technologies [36, 62, 74, 77], helping to make visualizations accessible to broader audiences [74].

Despite these benefits, the 36 critical papers highlight frustrations around the topic of interaction. The most frequent one is that in comparison to representation, interaction is rarely the focus of research efforts in visualization $[2,21,36,38,72,74,99,126]$. When interaction is the focus of research, the angle is often more on engineering or implementation than on designing for interaction $[36,99]$ and facilitating user analytic activities [2]. A key concern is the limited consideration for human (e.g., gaze, speech, haptics, sound, full-body) and technology modalities (e.g., pen, sketch, multi-touch surfaces), compared to the classic desktop-mouse-keyboard setups [36, 54, 65, 66, 74]. Beyond modalities, visualization systems are often not flexible when users want to express complex data queries $[16,54,66,120]$, integrate annotations [39,54], input new data [21], bookmark and extract insights [39], iterate over their activity history $[39,84]$, organize freely elements of the layout [54], choose their own statistical $[37,54]$ and visualization models $[54,79,100,102]$, and collaborate in real-time $[39,54]$.
This lack of flexibility stems from several factors, including technical challenges [90,102], costs [72] and the lack of theoretical foundations that bridge external representations with internal cognitive processes $[47,79,104]$. Two other factors are seemingly conflicting. On one hand, there is the failure of visualization systems to infer user goals. This is attributed to a strong focus from the community on data, tasks [14, 72] and domains [104] rather than on human goals; and to a difficulty to infer user goals via activity logs $[51,76,84]$. On the other hand, there is an overfocus on operationalizing user goals. As a result the visualization community ignores other objectives that broaden the interaction palette, such as engagement, playfulness and gamification [36, 39, 74], which reflect softer, or even lack of, user goal.

\subsection{How Visualization Conceptualizes Interaction}

We identified the need for more flexible interactions with respect to the outside world (e.g., environment, social aspect, modalities) and to the tool itself (e.g., user input, fluid interface permutation). We also identified the need to resolve the ambiguity between intent and lack of flexibility. Next, we review papers with a concept or a list tag to learn (i) how they conceptualize human intent, and (ii) how a visualization system can account for such intent to offer flexibility to the user.

\subsubsection{System, Task or Human?}

The concept and list papers reveal three approaches to conceptualize interaction for visualization: system-, task-, and human-centric.

System-centric approaches describe interaction in terms of operators $[20-22,28,120]$. These operators have been adapted to specific domains such as temporal visualizations [28] and visualizations of 2D data [99], and have been organized in a network [20] and in a hierarchy [22]. Focusing on system operations, system-centric approaches do not clarify neither the role of the user nor how the user interacts.

Task-centric approaches describe interaction in terms of low level tasks (also primitives or components) [2,99, 103]. Such low-level tasks have been put in relation with data types [106], means, target, cardinality, (temporal) order, and user roles (developers, authors, end users) [103]. It has been argued that high-level visualization tasks build on low-level interactions [66], but the relations and boundaries between low, intermediate, and high-level interactions are ill-specified [14,112].

Human-centric approaches describe interaction in terms of user intent $[32,39,47,99,126]$ rather than low-level mechanisms for supporting these intents. High-level cognitive activities introduce interactions such as notes (external source of data and external knowledge) and history that relate to insight actions [45], meta actions [45], and provenance actions [54], as opposed to exploration actions. Most human-centric approaches assume a high-level user intent in a data analysis context, such as making profitable investments with stock market data [45] or addressing the low productivity of a virtual factory network [97]). However, a few human-centric approaches expand the scope of user intent [79] by considering factors of interaction beyond data, system and tasks. Such factors include the environment (e.g., academic, museum), the context, the technology, the domain, the audience (e.g., web, analyst, casual), the task (e.g., exploration, immersion), and the properties of interaction (e.g., direct, embodied, aesthetic, rewarding) [36, 112]. Considering these factors builds higher-level views of interaction, that relate for example to mental models [79] and epistemic actions that take place during sense making, problem solving and decision making [104].

The broader the approach in terms of user intent, the less clear it is how a visualization system can operationalize the flexibility that is needed to accommodate this wider spectrum of user intents.

\subsubsection{Interaction \& Visualization Pipeline}

One of the most prominent concepts that describes a visualization system as a whole, from user's high level goal to low level data operations, is the visualization reference model, or visualization pipeline [16]. However, only 12/59 papers discuss the role of interaction in it. Most of those papers $[16,20,21,25,28,62,98]$ stress that interaction can affect all levels of the pipeline i.e., transformations of raw data, processed data, visual mappings and view. A few papers organize transformations [100] or interaction techniques [123] according to the level of the 
pipeline they affect. Others focus on specific interaction techniques, such as focus+context [70], selection [68] and distortion [68]; and some discuss high-level categories of interactions [65].

Attempts to describe interaction relative to the pipeline remain vague. Such statements explain that the closer an operator is to the view in the pipeline, the higher the possible amount of interaction [21]; that creating visualizations involves considering the visualization pipeline, characteristics of the data, and tasks to be performed [25]; or that the user can be an active participant controlling the pipeline at the different levels [112]. In fact, there is poor consistency between the user intent and the level of the pipeline the interaction occurs at (i.e. an intent perceived at a given level in the pipeline might technically affect another level) [62]. For example, techniques like filtering and dynamic queries have been classified as interactions occurring at the data level [123], although the user would be interacting with components (e.g., widgets) at the view level. The operator interaction framework [20,21] further sug gests that filtering can be an interaction at the view level (not affecting the underlying dataset) but also at the data level (affecting the underlying dataset). Also, as most of these frameworks [20-22, 62, 102, 120] are system-centric, they do not include user intent. In sum, the pipeline lacks the flexibility necessary to describing which interactions are available to a user, and does not clarify its connection to user intent.

\subsection{Summary of the Visualization View}

We found that in the field of visualization, interaction is conceptualized as a dialogue between a human user and the visualization system over a central object of interest: the data. But this dialogue has both low external flexibility (with respect to e.g., environment and modalities) and low internal flexibility (with respect to e.g., how modifiable visualization tools are). This dialogue also often limits user intentionality to data-analysis goals; and concepts advocating for a broader spectrum of user intentionality lack applicability. Making this dialogue successful demands systems flexible enough to accommodate broader user intents.

However, compared to the extensive research on visual perception, cognition, and semiotics that informs the design of effective visual representations, our community knows little about interaction design [99]. There is no agreement on even the basic terms being used to describe interaction. For example, a "task" refers interchangeably to a low-level task and a high-level goal. Or, the "means" of interaction refer interchangeably to the process carried out to achieve a task, or to the technical implementation that supports the task. Moreover, concepts and taxonomies of interaction essentially focus on data analysis objectives - thus overlook a larger scope of interactions. For example, few taxonomies (e.g. [14,54]) capture the interactions involved in authoring visualization, in creating data-driven narratives, or in inserting missing knowledge and external context. Most concepts and taxonomies still assume a setup with a single user (analyst) equipped with a mouse and keyboard and sitting in front of a computer. Most importantly, they do not help estimate the flexibility of visualization systems.

To address these limitations, we look next at the related field of HCI, in which interaction has its roots $[36,98,99,126]$. HCI considers interaction (the focus of user experience design) as a distinct concept from an interface (user interface design) $[6,98,99]$ to the extent that "humans use interfaces, but they experience interaction" [98].

\section{THE HCI VIEW OF INTERACTION}

A recent paper, What Is Interaction? [56] (that we call "the HCI review"), tries to answer a similar question to ours, for HCI. There is a need to establish what interaction is in visualization for the same reasons it is needed in HCI: the lack of definition of interaction hinders the development of a theory of interaction, which in turn results in folk notions, confusion, disagreements, and opinionated views on what interaction is, is not, or should be. We first summarize the HCI review. Then, we discuss how the view of interaction in HCI applies to visualization, before highlighting the differences between both fields.

\subsection{Seven Concepts that Characterize Interaction in $\mathbf{H C l}$}

The HCI review [56] extracts seven concepts of interaction. We summarize these concepts and specify how each formulates good interaction.
DIALOGUE: This concept is likely the most prominent from the early history of HCI. It sees interaction as a cycle of communication acts. Interaction is channeled through input and output from a computer perspective, and through perception and action from a user perspective. Based on Norman's action theory [85], users formulate a goal, then execute the actions needed to move that goal forward, perceive the output system state and relate that to their goal. Good interaction maximizes directness, simplicity and "naturalness" of the dialogue, while also providing "a strong sense of understanding and control" [56].

TRANSMISSION: This concept sees interaction as a transmission of information between the computer and the user. Based on information theory [40], the transmission rate is the amount of error-free information per time unit that is transferred over a communication channel in the presence of noise. Good interaction maximizes the transmission rate.

CONTROL: This concept sees interaction as an interactive minimization of error with respect to some user target. Based on control theory [60], interaction is continuous and defined via a system that consists of goals, inputs, outputs, feedback, feedforward and states. The user performs goal-driven actions, with feedback on the system state and feedfoward on its prospect state. Good interaction minimizes error and distance to user goal, and provides rapid and stable convergence to the target state. TOOL USE: This concept sees interaction as a series of tools that let a user interact with a computer. Tools are extensions of self like a hammer is a tool that one can manipulate to build. Based on activity theory [10] and the instrumental interaction model [5], a tool is a mediator between the user and an object of interest. Good interaction maximizes the usefulness or utility of such tool and amplifies user capabilities.

OPTIMAL BEHAVIOR: This concept sees interaction as an adaptive behavior under user capabilities, task, interface and environment constraints. It is based on the rationality paradigm [108] and adaptation theories such as information foraging [93] that state that humans tend to approximate optimal adaptation of their motor, perceptual and cognitive processes. Good interaction maximizes utility to the best of user capabilities within given constraints. Utility though does not equal efficiency. Sometimes wasting time is considered optimal, as "behavior that appears lazy or ignorant can be optimal for the organism to the extent it prefers saving effort over maximizing performance" [56].

EMBODIMENT: This concept sees interaction as the act of being and participating in the world, in which the users feel that the technology is an extension (or part) of them. Based on ecological psychology [34], embodiment focuses on the lived experience in which user's intention and actions are coupled with contexts (e.g., sitting on a chair, being tired). This differs from motor analysis, and with cognitive psychology (focusing on mental processing). Good interaction leverages artifacts that do not disrupt user pursuits and assist the user "in the wild".

EXPERIENCE: This concept sees interaction as a phenomenon shaped by user expectations, momentary reactions and recall. Based on user experience research [52], it places human experience as a key factor in how interaction unfolds, by leveraging aesthetics, emotion, fulfillment, surprise, stimulation, etc. The objective is non-utilitarian: it focuses on pleasure and meaning rather than performance and efficiency. Good interaction maximizes satisfaction of the user's psychological needs.

\subsection{Extending the View of Interaction in Visualization Through the Lens of $\mathrm{HCl}$}

We now discuss how the HCI concepts can extend the view of interaction in visualization we described in Sect. 3.

\subsubsection{Extending the Scope of User Intent}

Both $\mathrm{HCI}$ and visualization assume a teleological determination, i.e. that "human intentions postulate a norm or goal of interaction" [56]. The transmission concept assumes that the user has a message in mind, the tool a task to be accomplished, the optimal behavior defines intention as utility, the experience as a need to satisfy, and the other concepts include an explicit user goal. But user intent is not a requirement in HCI, that often aims for non-disruptive [34] or general-purpose tools that the user can appropriate depending on the task [5]. So while a good 
design should reveal how to use the tool (e.g., using affordances [87]), it should not impose what to do with it. Tools that support unforeseen user intents actually indicate successful interaction design [31].

Visualization includes user intent more explicitly, even within highlevel concepts (Sect. 3.1.1). Insight, or generation of new knowledge, is often said to be the ultimate goal of visualization [25,65]. While this might be true in a data analysis context, interaction with a visualization can have many goals. The goal might be to engage in your local community [9], to feel emotions through a representation of self [11], to reminisce about personal memories [111], to relate to 'humanized' data [33], or to experience a data-driven video [49]. In professional setups also, a decision maker might have some unanticipated goals such as to unruly remove visual information [29]. So, while an intent-driven perspective is more human-centric than a data- or task-driven one [14], extracting intent is not always feasible. We argue that interaction for visualization will benefit from expanding the scope of user intent and embracing intent-agnostic concepts [36]. As Dix summarized interaction design for appropriation, "You may not be able to design for the unexpected, but you can design to allow the unexpected" [31].

\subsubsection{Extending the Scope of User Profiling}

The user is a mandatory entity for interaction in both HCI and visualization. For HCI though, the spectrum of who is this user is broad; mainly any human being. That is because user profiling is a full stage of interaction design, with a variety of methods such as personas analysis [81], where designers construct various hypothetical archetypes narrating concrete details on each user's mental model, environment, skills, frustrations, attitudes, etc [42]. To foster designs that are easy to use while supporting complex needs, it is common that a novice and an expert persona co-exist [42]. In fact, HCI designs often promote a transition from novice to expert usage within the same tool [23], for example by revealing progressively interface complexity [107] or by providing visual guidance [71]. In contrast, the common approach to designing interactive systems in visualization is the design study [83] that establishes user profiles from the very beginning. Narrowing user profiles allows for a thorough characterization of a domain-specific problem and of the tasks to be supported. However, design studies tend to not consider user profiles from an interaction perspective (e.g., technology expertise, device, psychological state, environment, etc..).

In contrast to $\mathrm{HCI}$, the visualization research is more explicit about who the user is. According to Keefe [65], research on interaction in visualization differs from $\mathrm{HCI}$ in that it concerns "complex analysis tasks defined by a specific, highly motivated user population", particularly "scientists (or other domain experts)". This difference becomes less true with the emergence of casual [96] and personal [57] visualization research, as well as visualization democratization movements $[59,116]$ Yet, our review reveals that the profile of novice user is overlooked, as most papers refer to analysts and formal data analysis objectives when conceptualizing interaction. Visualization research also distinguishes designers and end-users when referring to data operations [22]. However, we saw in Sect. 3.3 that the roles of designers and end-users on the pipeline are ill-specified. The line between designer and end-user blurs even more with approaches that reduce the burden for programmatic generation of interactions $[59,100,102]$, placing custom visualizations in the hands of broader audiences [54].

Overall, dichotomies of users (i.e. analyst vs. non-analyst, expert vs. novice) likely contribute to shaping research sub-domains that are poorly connected to each other - in other words, contributes to poor external validity [12]. We argue that user profiles need not be binary, as visualization literacy is an often misunderstood metric [26, 27]. Also, many user profiles exist along the spectrum of expertise. Some analysts might experience novice needs such as a period of familiarization and sporadic usage of a tool; while "novices" may deal with complex tasks such as choosing a profitable real-estate investment among multiple trade-offs. We advocate for visualization research to adopt HCI methods to identify broader spectra of user profiles - even for a single tool - at the forefront of the design process.

\subsubsection{Extending the Scope of what Good Interaction is}

The most pronounced view of interaction both in visualization and in $\mathrm{HCI}$ is that of a dialogue, where human and computer communicate via action-reaction pairs. HCI further stresses that such dialogue should feel natural (e.g., using effective mapping [86] and quality feedback [94]) and be as direct and simple as possible (e.g., by comparing systems on interaction complexity using GOMS [18] or stochastic [3] models).

HCI concepts emphasize other desirable properties of interaction that can apply to visualization. The behavior concept suggests that visualization research could consider bounded rationality [44] contexts, in addition to strict normative rationality [30]. In some contexts, visualization users face massive cognitive overload under stress - for example decision makers that need to react real-time to tasks such as planning evacuation routes [113]. In such context, good interaction might equal simple interaction over minimum information, even at the cost of missing better alternatives [113]. Minimum information relates to the transmission concept, that is already considered when designing data representations (e.g., Tufte's data-ink-ratio [114]). This concept can also be applied to designing interaction. For example, the BIGNav technique for map navigation over uncertainty reduces user steps by progressively acquiring knowledge of the user's intent [78], instead of requiring the user to execute navigation commands.

Several HCI concepts are further overlooked in visualization, including user experience [36] and embodiment [74]. These relate to the notion of ubiquitous interaction, promoting interaction that supports multiple contexts (e.g., the field, the office, the home) while remaining consistent across them - as opposed to context-dependent interactions designed for point solutions [92,109]. To design for such environments, visualization can embrace concepts such as embedded data representations that integrate visual or even physical representations of data with the physical world [121], and design principles such as polymorphism for carrying interactive tools across different visualization systems [7].

\section{DEFINING INTERACTION FOR Visualization}

Although we saw that HCI can extend the view of interaction in visualization, determining the fundamental differences between the two fields is a longstanding challenge [35]. By comparing the visualization (Sect. 3) and the HCI (Sect. 4) views of interaction, we articulate these differences and propose a definition of interaction for visualization to encourage novel and more flexible interaction design practices.

\subsection{Key Differences in Interaction Between VIS and $\mathrm{HCl}$}

We identify key differences between visualization and HCI that are related to entities, focus, intent and flexibility of interaction:

ENTITIES AT PLAY: According to the HCI review, "The folk notion behind such uses [interaction] seems to be that of two entities (computer, human) engaged in an interplay of sorts." [56]. In visualization, this folk notion differs in that it must include a data entity. The entities engaged in an interplay of sorts would then be (data, computer, human), with the computer being a mediator between human and data.

PERFORMANCE FOCUS: Most HCI concepts aim for tools that are easy and intuitive to use with the objective to minimize effort, while maximizing performance. In contrast, visualization aims further at facilitating knowledge construction and sense-making [76]. Instead of minimizing effort, visualizations aim to engage users into thinking and reflecting on the data being explored [76]. Therefore, in contrast to the embodiment concept, constructing a mental model of a visualization can be essential [79]. Good interaction in that case may equal effort and seemingly slow interaction to get a deep understanding of the data. NATURE OF INTENT: We saw in Sect. 4.2.1 that the intent of users interacting with visualization can be softer than those related to formal data analysis - such as being related to emotions when interacting with artistic data-artifacts and engagement while interacting with a datadriven story. Yet even this broader notion of intent differs from intent related to general-purpose HCI tool in that it is related to data. While HCI systems might contain data, visualization by definition is a data interface that exposes a human to an intangible data source. Therefore, intent in visualization can be refined as being a data-related intent. 
INTENT INTEGRATION: The HCI review indicates that in HCI it is often assumed that users form their goals and intentions outside of the interaction cycle. For example, a person might have the goal to order food online, and HCI concepts will help make the interaction with a computer better to make this goal of ordering food efficient, satisfying, or a unique experience. The HCI review calls it a blind spot in HCI research: "while all concepts subscribe to teleological reasoning, most say little about how intentions are formed or affected by interaction". In visualization, this is clearly not the case. Data exploration is studied as an iterative process that takes many steps, during which goals and intentions evolve based on views that are updated through interaction.

FLEXIBILITY WITHIN-THE-TOOL: We saw in Sect. 4.2.3 that the HCI concepts can expand the flexibility of a tool within the outside world (e.g., not to disrupt other activities with the embodiment concept) as well as within our internal world (i.e. to satisfy psychological needs with the experience concept). Visualization differs in that it requires flexibility within the tool itself. Interacting with a visualization often results in changing tasks on the fly, to iteratively build an understanding of the data through a series of smaller data questions. So while with HCI the user would mostly interact at the view level of the visualization pipeline, with visualization they might need to interact with all levels of the pipeline. This means that the user likely benefits from having access to a large variety of means of interacting with the visualization.

To summarize, by comparing the visualization and HCI literature we extend the view of interaction in visualization in terms of: scope of user intentionality, user profiling, and our notion of what good interaction means - especially with respect to the external world. Yet some questions cannot be answered by the HCI literature, mainly because HCI lacks an explicit tie to the data component. Specifically, it is unclear how to conceptualize interaction when (i) there is a datarelated user intent and (ii) there is a need for interaction flexibility within-the-tool with respect to that intent. We address the first point about data-related intent next, through our definition of interaction. We will clarify the second point about flexibility within-the-tool in Sect. 6.

\subsection{What is Interaction for Visualization?}

Our definition of interaction for visualization is based on two assumptions: i) interactions with visualizations are a subset of the interactions with interactive systems - thus we do not capture all interactions captured by the HCI concepts; and ii) interactions with interactive systems are a subset of interactions with people's own mind and environment thus our definition does not capture all interactions people might perform while exposed to interactive systems. By comparing findings from the visualization (Sect. 3) and the HCI (Sect. 4) literature, we synthesize the following compact definition of interaction for visualization:

Interaction for visualization is the interplay between a person and a data interface involving a data-related intent, at least one action from the person and an interface reaction that is perceived as such.

This definition consists of the following mandatory interaction components (i.e. describing interaction requires all of these components):

INTERPLAY: We frame the definition via a dialogue metaphor as it is the common schema we observed in both visualization and HCI. The word "dialogue" itself though is an overloaded term in HCI, which might be associated with interfaces that work sequentially, like human-to-human communication. Therefore we use its synonym word interplay.

PERSON: We replace the commonly reported entity of human "user" with the term person, that is more likely to be perceived as genderneutral [13]. Although we assume that the person is a human being, the term does not exclude non-human entities [110]. Thus we do not exclude mixed initiatives [53] nor humans becoming "data interfaces".

DATA INTERFACE: A key concern in visualization was the limited use of human and technology modalities. To broaden the modality scope, we call the visualization system entity data interface. This allows for a technology-agnostic perspective including non-computerized visualization such as physicalizations, infographics and bio-inspired visualizations [89]. We do not specify the modality (e.g, "visual") as many modalities might apply [91], such as sonification and tactilization (described by Card et al. as "perceptualization" [16]). The data interface is a system containing a set of controls that are perceivable by the person, and linked to a data entity. The data interface can contain other entities (e.g., a computer). The data entity itself is an abstracted intangible source that, like other abstracted notions such as "knowledge", cannot physically constitute an actor in a two-way communication. Technically, the person interacts with the data interface (not the data entity), as the data interface is the one capable of providing a response.

ACTION: We adopt the term action performed by the person without constraining its granularity (e.g., it can be a low or a high level action). We include any action, physical or mental, and regardless of modality, that causes the reaction of the data interface.

ACTION-REACTION: Visualization and HCI agree that the interaction dialogue requires that the interface responds to a person's action. While such action-reaction does not limit to a specific modality, (e.g. touch, or thought), it is a cause-effect relationship. Consider two people A and B separated by a wall. If A speaks and B does not respond verbally, this is not a dialogue. Now, A and B are in the same room, B still does not respond verbally but A sees B smile. There was a response to the action, regardless of the modality being used (voice or facial expression).

REACTION PERCEIVED AS SUCH: Visualization and HCI agree that the interaction dialogue requires that the person perceives the reaction of the data interface. If $\mathrm{A}$ and $\mathrm{B}$ are separated by a wall and $\mathrm{B}$ responds too quietly to be heard by $\mathrm{A}$, then the reaction is not perceived. If $\mathrm{B}$ responds loudly but in an incomprehensible way, the reaction is perceived, although the dialogue is a bad one. We saw that the perception of the reaction is constrained by a time entity. Time expectations can differ depending on the context [16]. For example, a system that requires a long time to compute a statistical model can provide a timely reaction even if it is not immediate. Therefore, we refrain from using the common descriptors of "rapid" or "immediate" reaction. Timeliness is only one factor to the person perceiving the reaction as a response to their action. In a broader sense, for a dialogue to occur the person must perceive the causality of their action (i.e., self-agency [62]). To capture causality, we specify that the reaction must be perceived as such.

DATA-RELATED INTENT: Visualization and HCI agree that interaction involves a teleological determination (i.e. a norm or goal of interaction). What differs is that the visualization intent (Sect. 5.1) is tied to a data source and is formed iteratively within the interaction cycle. We use the term data-related intent to stress this difference. We do not use a term such as "data-oriented intent" to not enforce interaction as a goal-driven activity but include implicit interaction [61] and coarsely approximated intent [74]. We also do not enforce the temporality of the intent (i.e., it can be formed in advance of the interaction or not). We also do not enforce who the beholder of the data-related intent is. Although the beholder is usually the person, this leaves open the possibility that the beholder of the intent be the designer of the data interface.

To further highlight the boundaries of our definition, we now answer the question "Is this an interaction for visualization?" for six scenarios.

SCENARIO 1: Joe is staring at a visualization without performing any action that is interpretable by the visualization. He is reasoning (mentally) about the visualized data. There are further (computer initiated) animations, real time changes that show important information that Joe takes into account in his reasoning process.

Answer: No. While there were several actions from the person, the visualization did not return any reaction as a response to those actions.

SCENARIO 2: Joe wants to focus on a cluster of data points. Using a mouse, he traces a lasso to select the data points. The visualization draws the lasso with the exact same color as the background. Answer: No. While there was an action-reaction, the reaction of the system was not perceived by the person.

SCENARIO 3: Joe notices a slider widget. He intends to filter out some data points. He moves the slider and the visualization is zoomed-in. He does not understand what is going on.

Answer: Yes. Because the reaction of the visualization (although not understood) was perceived by the person. 
SCENARIO 4: An airport provides a visualization that updates automatically to show all departure gate data. Jack looks for his gate number, but the visualization changes too quickly. A gaze detector tracks Jack's unfocused gaze attempts and slows down the rate at which the visualization changes. Jack now locates his gate number.

Answer: Yes. There was an action-reaction perceived by the person. Such gazing action was not interpretable by the interface in scenario 1 .

SCENARIO 5: Averell cannot remember where he parked his car. In the parking a screen shows car $\mathrm{CO} 2$ emission data to motivate car drivers to use their car less. Averell is not careful and he bumps his head on the screen. Because the screen is touch-enabled, this contact rearranges the data points. Averell sees some graphics moving around but does not pay attention. He ignores the screen and continues his search.

Answer: No. While there was an action-reaction perceived by the person, there was no data-related intent (unless the designer, having such intent, had caused Averell to non-consciously process some data).

SCENARIO 6: Like Averell, William bumps his head on the display. But when he sees the graphics move around he gets curious and starts touching the display to explore what these graphic things are about.

Answer: Yes. Although the interaction was not initiated by an explicit intent, there was an implicit data-related intent involved in the process.

\section{Flexible Data Interfaces}

Definitions are essential to establish foundational knowledge in any applied domain. But it is also critical to make definitions actionable through applicable concepts. Our definition provides several dimensions that the visualization community can investigate to enrich interactivity in data interfaces (e.g., action, reaction, perception, intent). In this section, we provide one example of making the definition actionable by focusing on how to enrich the actions in data interfaces.

Our review of visualization research stressed the need for more flexible interfaces in visualization (Sect. 3); while we also saw that the HCI concepts do not promote flexibility-within-the-tool - which is essential for visualization (Sect. 5.1). Here we discuss how the notion of allowable actions offered by a data interface can be used to enrich its flexibility. We first propose a classification of possible actions that we then use to operationalize the flexibility of a data interface. Then we present examples of how the concept of flexibility can help to practically describe and compare data interfaces.

\subsection{Classifying Actions}

Among the different levels of granularity of actions $[14,62,103]$, we focus on intermediate-level actions [62] because: i) they have a datarelated meaning thus can support complex cognitive activities, in contrast to low-level interactions such as mouse clicks; and ii) they are operationalizable, in contrast to high-level interactions [104].

Such data-level actions are commonly described using the visualization pipeline schema [62]. However, Sect. 3.3 revealed that i) the pipeline makes it difficult to describe interaction from a system-centric perspective (e.g., propagation phenomena [62]); and that ii) its relation to the person's intent is ambiguous (e.g., conflicted operation stages [21]). To avoid such problems, we adopt a human-centric interpretation of the pipeline. We replace the system-centric interpretation with a semantic one from a person's perspective. Particularly, our categorization is based on which are the person's allowed actions within the data interface to achieve their intents. By merging this semantic interpretation of the pipeline with related taxonomies [45, 54, 62], we consider three categories of allowable actions within a data interface: data actions, perceptualization data actions, and non-data actions.

DATA ACTIONS operate on the intangible data entity ("data change" [45]). They can be input data actions or processing data actions.

- Input data actions operate on raw data values. Examples include: edit, update, modify and correct data [62]; or add, create, insert and import data or meta data. These actions can be basic, e.g., importing a dataset in the system [21], sophisticated, e.g., inputting data or external knowledge $[4,118]$ through creating data-aware annotations [54]), and can be disregarded, e.g., collecting data [62].
- Processing data actions apply functions/transformations to the input/raw data. Examples include: compile, filter, select subset, and query data [54]. These actions can be used to transform data "into a form that is suitable for visualization" [62], to derive from the raw data (e.g., normalized values, statistical summaries and aggregates [54]), and to change a statistical model or its parameters [37].

PERCEPTUALIZATION DATA ACTIONS operate on the perceptual formatting of the data entity ("visual change" [45]). They can be mapping data actions or presentation data actions.

- Mapping data actions assign data entities to perceptual marks and variables [62], and layout/rearrange the data. These actions constitute the abstract presentation form of the representation [62].

- Presentation data actions operate on the specific presentation of the data [62]. Examples include: mark something as interesting, navigate (e.g., pan and zoom) [45]), stylize, highlight, and decorate [62].

NON-DATA ACTIONS do not operate explicitly on the data nor on the data presentation [45], but on interaction entities not captured by the pipeline model. They can be meta, social or interface actions.

- Meta actions operate on the person's own actions [45]. Examples include: undo, redo, record activity, log, and change history [45].

- Social actions connect the person to other persons. Examples include: communicate and discuss with peers, share results, and coordinate the data analysis work of multiple groups [54].

- Interface actions operate on other data interface components. Examples include: take notes that are not bound to data items (e.g., document observations/questions/hypotheses/reflections/goals [54]), manage knowledge [45], externalize thoughts [117], and organize, open, close, maximize, and layout/rearrange the interface [54].

\subsection{Operationalizing Flexibility}

Several properties of allowable actions (e.g., complementarity, fitness, genre, diversity, and flexibility [105]) can describe the quality of interaction within a data interface. Here we focus on diversity and flexibility.

As diversity, we consider the given interaction means: the set of distinct controls that the data interface provides to the person for performing actions. Interaction means range from basic to complex, thus we do not use the term "interaction technique" that is often associated to elaborated means. An action is allowable only if the data interface provides at least one interaction means for that action. There can be more than one interaction means per action (e.g., sort data points by: clicking on a button, speaking a voice command, or rearranging data points manually). An interaction means can be characterized according to its cardinality, human modality, and technology modality.

As flexibility, we consider concept that is broader than diversity and that builds on our data-oriented interpretation of Gibson's concept of affordance [43] (it differs from the affordance we saw in Sect. 4.2.1 by Norman [87]). In Gibson's terms, "affordance is what the environment offers the individual" [43]. The set of affordances depends on the object (the data interface) and the person (i.e., a chair affords sitting for an adult, but not for a baby). By offering more interaction means, a flexible data interface can bolster diversity of affordances, which in turn can help design for inter-individual differences and accessibility. We conflate our classification of allowable data-level actions (what the person can do with the data) with the concept of interaction means (how the person can perform these actions) to define flexibility as follows:

Flexibility within a data interface is the number of distinct, allowable actions of a person on the interface, as well as the number of interaction means with which the person can perform each action.

This operationalization of flexibility makes it possible to describe and compare data interfaces. The purpose is not to plainly count features, but to provide a simple and actionable way of describing interactive visualization systems. Indeed more interactions does not mean better interface, as more interactions than necessary have a cost [72,115]. Next we show through two examples how to compare the flexibility of existing tools using i) the cardinality of interaction means and ii) the cardinality of allowable actions based on their category. 
Category - Perceptualization data actions: we compare Dust \& Magnet [127] with its physical version [73] ("Zooids").

\section{INTERACTION MEANS}

\begin{tabular}{|c|c|c|c|}
\hline \multirow{2}{*}{\multicolumn{2}{|c|}{ ACTION }} & & \\
\hline & & Dust \& Magnet & Zooids \\
\hline \multirow{5}{*}{ 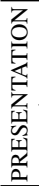 } & Mark data & 1) point with finger, & 1) point with finger, \\
\hline & point as & 2) move mouse cursor on & 2) stick object besides, \\
\hline & interesting & top, 3) stick object besides & 3) put empty glass upside-down over \\
\hline & Highlight & 1) click on*, 2) touch* & not implemented \\
\hline & data point & *highlights the outline of th & point and shows its label \\
\hline \multirow{5}{*}{ 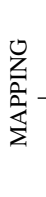 } & Rearrange & 1) create magnet* & 1) create magnet*2) put points in boxes \\
\hline & data points & & labelled with names of dimensions \\
\hline & *attracts $P$ & oints basea on inetr vames. & or the dimension assigned to the magnet \\
\hline & Encode & not implemented & 1) map dimension to color using paint, \\
\hline & dimension & & $\begin{array}{l}\text { 2) map dimension to sticker shape, color, } \\
\text { texture, size, etc.. and stick on data points }\end{array}$ \\
\hline
\end{tabular}

Both systems allow for three actions. Dust \& Magnet offers more interaction means for highlighting data points (presentation data action); and Zooids offers more for both mapping data actions. This example also stresses that the same action can be performed for different interactions. Putting an empty glass upside-down over a data point, marks the data point as interesting. But this action could also be a data-aware annotation (input data action) because if the Zooid moved, the glass, if lightweight, would move with it. This action, as well as painting on, or adding stickers to Zooids, illustrate that high flexibility in interaction means allows for appropriation of a data interface.

Category - Data actions: we compare the HomeFinder [122] and the constructive visualization approach [59] colored tiles ("Token").

\section{INTERACTION MEANS}

\begin{tabular}{|c|c|c|c|}
\hline \multirow{2}{*}{\multicolumn{2}{|c|}{ ACTION }} & \multirow{3}{*}{$\begin{array}{l}\text { HomeFinder } \\
\text { 1) move slider widget, 2) drag } \\
\text { an icon to a certain location }\end{array}$} & \multirow{3}{*}{$\begin{array}{r}\text { Token } \\
\text { not implemented }\end{array}$} \\
\hline & & & \\
\hline 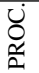 & Make data query & & \\
\hline \multirow{3}{*}{ 占 } & Add data point & not implemented & 1) add token \\
\hline & Correct data point & not implemented & 1) replace, 2) recolor token \\
\hline & $\begin{array}{l}\text { Add metadata to } \\
\text { data point }\end{array}$ & not implemented & $\begin{array}{l}\text { 1) annotate token, 2) annotate } \\
\text { surrounding background }\end{array}$ \\
\hline
\end{tabular}

The first example focused on cardinality of interaction means. This example focuses on cardinality of allowable actions as a way of revealing what interactions might be lacking in existing tools. For example, HomeFinder allows for data processing actions while Token does not. On the other hand, Homefinder does not support the input actions that Token does. However, a real-estate agent having information about a condo and wanting to update the data, or a potential buyer having visited a house and wanting to add metadata to it, are credible data input scenarios that a more flexible system could support.

\section{Research Opportunities \& Challenges}

Broadening the scope of interaction comes with research opportunities and challenges for visualization. Increasing the cardinality of allowable interactions does not necessarily improve the quality of interaction in a data interface. Improving the quality of interaction also requires supporting larger numbers of easy to use and fluent [36] interaction means and providing elaborated interaction techniques [38], while considering the environment $[63,73,121]$, leveraging human and technology modalities [54,65, 66,74], and accounting for a broad spectrum of user profiles $[57,59,96,116]$. Interaction also has a cost [72]. Similarly to the savvy concept of Tufte's data-ink-ratio for visual representations [114], interaction design needs to specify its own boundaries [67]. For example, offering too many allowable actions may result in increased interface complexity and distract from thinking and reflection.

Our categorization of interactions stresses several research challenges. Perceptualization actions stress the challenge of empowering the person to form their own visual models (e.g., through authoring $[69,119]$ and free-form sketching $[75,124])$, by creating repre- sentations that match their reasoning models [79, 104]. Processing actions stress the challenges of supporting complex data queries [54] and of merging statistical modelling and data exploration within a single interface [37]. Non-data actions stress the challenge of fostering collaboration [55,80] and reflection over past activities [39, 50, 84]. Input actions - perhaps the most overlooked actions [62] - stress the challenge of enriching interaction means for data input [4, 26, 62], ranging from correcting erroneous data [82] to adding metadata and data-aware annotations $[39,54,117]$ to creating knowledge from scratch $[59,111]$. From a broader perspective, input actions are the actions that allow to refine and integrate human knowledge into the data interface [118].

\section{LimitaTions}

We do not claim that our definition captures all perspectives of interaction. First and foremost, our definition is one among many possible definitions. For example, interactions with absence of intent are a perspective that our definition does not capture.

Second, our critical review is not comprehensive. Some important perspectives (e.g., on interaction directness [15] and on user engagement [58]) are left out due to limitations both of the expert input and of our paper selection method. For example, papers about interaction techniques were often not suggested by experts as they are not framed and recalled as defining interaction; discarding non-refereed publications such as PhD theses likely results in excluding some controversial work; and the response rate to our questionnaire was relatively small $\sim 35 \%$ (22/64), with senior researchers responding more often than junior researchers. Further, our methodology does not include the perspectives of practitioners, both professional and incidental.

Fourth, the flexibility concept that focuses on allowable actions is only one way of making our definition actionable. Therefore we see this definition as a thinking tool that the community can use to explore other ways of reasoning about interaction in visualization. For example, one could focus on the "reaction" of the data interface (e.g., how different types of user feedback can enrich the quality of interaction).

\section{Conclusions}

Our methodology for critical reviews that incorporates human expertise and quantitative metrics allowed us to analyze (i) mandatory and optional components of interaction as well as its properties, (ii) the benefits and critiques on the interaction topic, and (iii) the current conceptualization of interaction in visualization. By comparing our findings from the visualization literature with the view of interaction from the HCI literature, we identified opportunities for broadening the view of interaction in visualization on (i) user intentionality, (ii) user profiling, and (iii) what good interaction is. That comparison led us to distill the fundamental differences between visualization and HCI that are related to entities, focus, intent and flexibility of interaction.

Building on these findings, we gave one answer to the question "what is interaction for data visualization?" through a compact definition that captures human intent and interaction causality, is technology agnostic, and includes the full spectrum of human senses.

Finally, our operationalization of the notion of within-the-tool flexibility is an example of making this definition actionable. By categorizing interactions based on data-level actions and intents in relation to the visualization pipeline, we provide a systematic approach for describing and comparing visualization systems. This operationalization can aid visualization designers estimate how modifiable a visualization system is, and how diverse the palette of available interactions is.

We hope that our inclusive view of interaction will unify the visualization community under a common goal: the creation of visualization systems that empower those who use them.

\section{ACKNOWLEDGMENTS}

We thank P. Dragicevic, G. Bailly, B. Shneiderman, J-D. Fekete, E. Chi, M. McGuffin, T. Munzner, J. Heer, N. Elmqvist, P. Parsons, M. Tory, M. Brehmer, S. Feiner, H. Siirtola, P. Isenberg, as well as the anonymous contributors of the survey for their valuable input. 


\section{REFERENCES}

[1] R. S. Amant. Natural interaction with visualization systems. Internet Comput., 19(6):60-64, 2015.

[2] R. Amar, J. Eagan, and J. Stasko. Low-level components of analytic activity in information visualization. In Proc. InfoVis, pp. 111-117, 2005.

[3] J. R. Anderson. ACT: A simple theory of complex cognition. Am Psychol, 51(4):355-365, 1996.

[4] T. Baudel. From information visualization to direct manipulation. In Proc. UIST, pp. 67-76, 2006.

[5] M. Beaudouin-Lafon. Instrumental interaction: an interaction model for designing post-WIMP user interfaces. In Proc. CHI, pp. 446-453, 2000

[6] M. Beaudouin-Lafon. Designing interaction, not interfaces. In Proc. AVI, pp. 15-22, 2004.

[7] M. Beaudouin-Lafon and W. E. Mackay. Reification, polymorphism and reuse. In Proc. AVI, pp. 102-109, 2000.

[8] R. A. Becker and W. S. Cleveland. Brushing Scatterplots. Technometrics, 29(2):127-142, 1987.

[9] J. Bird and Y. Rogers. The pulse of tidy street: Measuring and publicly displaying domestic electricity consumption. In Proc. Pervasive Workshop, 2010.

[10] S. Bødker. Through the interface - a human activity approach to user interface design. DAIMI Report Series, 16(224), 1987.

[11] F. Botros, C. Perin, B. A. Aseniero, and S. Carpendale. Go and grow: Mapping personal data to a living plant. In Proc. AVI, pp. 112-119, 2016.

[12] G. H. Bracht and G. V. Glass. The external validity of experiments. Am. Educ. Res. J., 5(4):437-474, 1968.

[13] A. Bradley, C. MacArthur, M. Hancock, and S. Carpendale. Gendered or Neutral? Considering the Language of HCI. In Proc. GI, pp. 163-170, 2015.

[14] M. Brehmer and T. Munzner. A multi-level typology of abstract visualization tasks. TVCG, 19(12):2376-2385, 2013.

[15] S. Bruckner, T. Isenberg, T. Ropinski, and A. Wiebel. A Model of Spatial Directness in Interactive Visualization. TVCG, pp. 2514-2528, 2018.

[16] S. K. Card, J. D. Mackinlay, and B. Shneiderman. Readings in information visualization: using vision to think. Morgan Kaufmann, 1999.

[17] S. K. Card, T. P. Moran, and A. Newell. The keystroke-level model for user performance time with interactive systems. Commun. ACM, 23(7):396-410, 1980.

[18] S. K. Card, A. T. Newell, and T. P. Moran. The Psychology of HumanComputer Interaction. CRC Press, 1983.

[19] S. K. Card, G. G. Robertson, and J. D. Mackinlay. The information visualizer, an information workspace. In Proc. CHI, pp. 181-186, 1991.

[20] E. Chi. A taxonomy of visualization techniques using the data state reference model. In Proc. InfoVis, pp. 69-75, 2000.

[21] E. Chi and J. Riedl. An operator interaction framework for visualization systems. In Proc. InfoVis, pp. 63-70, 1998.

[22] M. Chuah and S. Roth. On the semantics of interactive visualizations. In Proc. InfoVis, pp. 29-36, 1996.

[23] A. Cockburn, C. Gutwin, J. Scarr, and S. Malacria. Supporting novice to expert transitions in user interfaces. Comput Surv, 47(2):1-36, 2014

[24] J. Cohen. A coefficient of agreement for nominal scales. Educ. Psychol. Meas., 20(1):37-46, 1960.

[25] K. A. Cook and J. J. Thomas. Illuminating the Path: The Research and Development Agenda for Visual Analytics. IEEE Press, 2005.

[26] M. Correll. Ethical dimensions of visualization research. In Proc. CHI, pp. 188:1-188:13, 2018

[27] M. Correll. What does "visualization literacy" mean, anyway? - Multiple Views: visualization research explained, 2018.

[28] C. Daassi, L. Nigay, and M.-C. Fauvet. A taxonomy of temporal data visualization techniques. I3, 5(3):41-63, 2005.

[29] E. Dimara, G. Bailly, A. Bezerianos, and S. Franconeri. Mitigating the attraction effect with visualizations. TVCG, 25(1):850-860, 2019.

[30] E. Dimara, S. Franconeri, C. Plaisant, A. Bezerianos, and P. Dragicevic. A task-based taxonomy of cognitive biases for information visualization. TVCG, 2019.

[31] A. Dix. Designing for appropriation. In Proc. BCS-HCI, pp. 27-30, 2007.

[32] A. Dix and G. Ellis. Starting simple : Adding value to static visualisation through simple interaction. In Proc. AVI, pp. 124 - 134, 1998.

[33] J. Donath, A. Dragulescu, A. Zinman, F. Viégas, and R. Xiong. Data portraits. In SIGGRAPH Art Gallery, pp. 375-383, 2010.

[34] P. Dourish. Where the action is: the foundations of embodied interaction.
MIT Press, 2004.

[35] A. Ebert, G. Veer, G. Domik, N. D. Gershon, and I. Scheler, eds. Building Bridges: HCI, visualization, and non-formal modeling. Springer, 2014

[36] N. Elmqvist, A. V. Moere, H.-C. Jetter, D. Cernea, H. Reiterer, and T. Jankun-Kelly. Fluid interaction for information visualization. Inf. Vis., 10(4):327-340, 2011.

[37] A. Endert, P. Fiaux, and C. North. Semantic interaction for visual text analytics. In Proc. CHI, pp. 473-482, 2012.

[38] J.-D. Fekete. Advanced interaction for information visualization. In Proc. PacificVis, 2010

[39] A. Figueiras. Towards the understanding of interaction in information visualization. In Proc. IV, pp. 140-147, 2015.

[40] P. M. Fitts. The information capacity of the human motor system in controlling the amplitude of movement. J. Exp. Psychol., 47(6):381-391, 1954.

[41] J. L. Fleiss, B. Levin, and M. C. Paik. Statistical methods for rates and proportions. John Wiley \& Sons, 2003.

[42] E. Friess. Personas and decision making in the design process. In Proc. CHI, pp. 1209-1218, 2012.

[43] J. J. Gibson. The theory of affordances. Hilldale, USA, 1:2, 1977.

[44] G. Gigerenzer and R. Selten. Bounded rationality: The adaptive toolbox. MIT press, 2002.

[45] D. Gotz and M. X. Zhou. Characterizing users' visual analytic activity for insight provenance. Inf. Vis., 8(1):42-55, 2009.

[46] M. J. Grant and A. Booth. A typology of reviews: an analysis of 14 review types and associated methodologies. Health Info Libr J, 26(2):91-108, 2009.

[47] H. Guo, S. R. Gomez, C. Ziemkiewicz, and D. H. Laidlaw. A case study using visualization interaction logs and insight metrics to understand how analysts arrive at insights. TVCG, 22(1):51-60, 2016

[48] R. B. Haber and D. A. Mcnabb. Visualization idioms: A conceptual model for scientific visualization systems. VISC, pp. 74-93, 1990.

[49] N. Halloran. The Fallen of World War II. http://www. fallen.io/ww2/.

[50] Y. Han, G. D. Abowd, and J. Stasko. Flexible organization, exploration, and analysis of visualization application interaction events using visual analytics. In Proc. VIS Workshop, 2016.

[51] L. Harrison, W. Dou, A. Lu, W. Ribarsky, and X. Wang. Analysts aren't machines: Inferring frustration through visualization interaction. In Proc. VAST, pp. 279-280, 2011.

[52] M. Hassenzahl and N. Tractinsky. User experience - a research agenda. $B \& I T, 25(2): 91-97,2006$.

[53] C. Healey, S. Kocherlakota, V. Rao, R. Mehta, and R. St. Amant. Visual perception and mixed-initiative interaction for assisted visualization design. TVCG, 14(2):396-411, 2008.

[54] J. Heer and B. Shneiderman. Interactive dynamics for visual analysis. Comm. of the ACM, 55(4):1-26, 2012.

[55] J. Heer, F. B. Viégas, and M. Wattenberg. Voyagers and voyeurs: supporting asynchronous collaborative information visualization. In Proc. CHI, pp. 1029-1038, 2007.

[56] K. Hornbaek and A. Oulasvirta. What is interaction? In Proc. CHI, pp. 5040-5052, 2017

[57] D. Huang, M. Tory, B. Adriel Aseniero, L. Bartram, S. Bateman, S. Carpendale, A. Tang, and R. Woodbury. Personal visualization and personal visual analytics. TVCG, 21(3):420-433, 2015.

[58] Y. Hung and P. Parsons. Assessing User Engagement in Information Visualization. In Proc. CHI EA, pp. 1708-1717, 2017.

[59] S. Huron, S. Carpendale, A. Thudt, A. Tang, and M. Mauerer. Constructive visualization. In Proc. DIS, pp. 433-442, 2014

[60] R. J. Jagacinski and J. M. Flach. Control theory for humans: quantitative approaches to modeling performance. CRC Press, 2003.

[61] L.-E. Janlert and E. Stolterman. The meaning of interactivity-some proposals for definitions and measures. Hum.-Comput. Interact., 32(3):103138,2017

[62] Y. Jansen and P. Dragicevic. An interaction model for visualizations beyond the desktop. TVCG, 19(12):2396-2405, 2013

[63] Y. Jansen, P. Dragicevic, P. Isenberg, J. Alexander, A. Karnik, J. Kildal, S. Subramanian, and K. Hornbæk. Opportunities and challenges for data physicalization. In Proc. CHI, pp. 3227-3236, 2015.

[64] W. Javed and N. Elmqvist. Stack zooming for multi-focus interaction in time-series data visualization. In Proc. PacificVis, pp. 33-40, 2010.

[65] D. Keefe. Integrating visualization and interaction research to improve scientific workflows. $C G \& A, 30(2): 8-13,2010$.

[66] D. Keefe and T. Isenberg. Reimagining the scientific visualization inter- 
action paradigm. Computer, 46(5):51-57, 2013.

[67] M. Keehner, M. Hegarty, C. Cohen, P. Khooshabeh, and D. Montello. Spatial reasoning with external visualizations: What matters is what you see, not whether you interact. Cognitive Science, 32(7):1099-1132, 2008.

[68] D. Keim. Information visualization and visual data mining. TVCG, 8(1):1-8, 2002.

[69] N. W. Kim, E. Schweickart, Z. Liu, M. Dontcheva, W. Li, J. Popovic, and H. Pfister. Data-driven guides: supporting expressive design for information graphics. TVCG, 23(1):491-500, 2017.

[70] R. Kosara, H. Hauser, and D. L. Gresh. An interaction view on information visualization. In Proc. Eurographics, pp. 123-137, 2003.

[71] G. Kurtenbach and W. Buxton. The limits of expert performance using hierarchic marking menus. In Proc. CHI, pp. 482-487, 1993.

[72] H. Lam. A framework of interaction costs in information visualization. TVCG, 14(6):1149-1156, 2008.

[73] M. Le Goc, C. Perin, S. Follmer, J.-D. Fekete, and P. Dragicevic. Dynamic composite data physicalization using wheeled micro-robots. TVCG, 25(1):737-747, 2019.

[74] B. Lee, P. Isenberg, N. H. Riche, and S. Carpendale. Beyond mouse and keyboard: expanding design considerations for information visualization interactions. TVCG, 18(12):2689-2698, 2012.

[75] B. Lee, R. H. Kazi, and G. Smith. SketchStory: Telling more engaging stories with data through freeform sketching. TVCG, 19(12):2416-2425, 2013.

[76] H.-N. Liang, P. C. Parsons, and H.-C. Wu. An exploratory study of interactivity in visualization tools. JILR, 21(1):5-45, 2010.

[77] Lingyun Yu, P. Svetachov, P. Isenberg, M. H. Everts, and T. Isenberg. FI3D: Direct-touch interaction for the exploration of 3D scientific visualization spaces. TVCG, 16(6):1613-1622, 2010.

[78] W. Liu, R. L. D’Oliveira, M. Beaudouin-Lafon, and O. Rioul. BIGnav:Bayesian information gain for guiding multiscale navigation. In Proc. CHI, pp. 5869-5880, 2017.

[79] Z. Liu and J. T. Stasko. Mental models, visual reasoning and interaction in information visualization: A top-down perspective. TVCG, 16(6):999$1008,2010$.

[80] N. Mahyar and M. Tory. Supporting communication and coordination in collaborative sensemaking. TVCG, 20(12):1633-1642, 2014

[81] N. Marsden and M. Pröbster. Personas and Identity. In Proc. CHI, pp. 1-14. New York, New York, USA, 2019.

[82] N. Mccurdy, J. Gerdes, and M. Meyer. A framework for externalizing implicit error using visualization. TVCG, 25(1):925-935, 2019.

[83] T. Munzner. A nested model for visualization design and validation. TVCG, 15(6):921-928, 2009.

[84] M. Nafari and C. Weaver. Query2Question: Translating visualization interaction into natural language. TVCG, 21(6):756-769, 2015.

[85] D. Norman. User centered system design. CRC Press, 1986.

[86] D. Norman. Designing interaction. chap. Cognitive artifacts, pp. 17-38. 1991.

[87] D. Norman. The design of every day things. Basic Books, 2013.

[88] B. J. Oates. Researching information systems and computing. Sage, 2005.

[89] N. Oxman. Variable property rapid prototyping. Virtual Phys. Prototyp., 6(1):3-31, 2011.

[90] G. Palmas, M. Bachynskyi, A. Oulasvirta, H.-P. Seidel, and T. Weinkauf. MovExp: A versatile visualization tool for human-computer interaction studies with 3D performance and biomechanical data. TVCG, 20(12):2359-2368, 2014.

[91] B. Patnaik, A. Batch, and N. Elmqvist. Information olfactation: Harnessing scent to convey data. TVCG, 25(1):726-736, 2019.

[92] W. A. Pike, J. Stasko, R. Chang, and T. A. O'Connell. The science of interaction. Inf. Vis., 8(4):263-274, 2009.

[93] P. Pirolli and S. Card. Information foraging. Psychol. Rev., 106(4):643675, 1999.

[94] H. Pohl and R. Murray-Smith. Focused and casual interactions. In Proc. CHI, p. 2223, 2013.

[95] M. Pohl, S. Wiltner, and S. Miksch. Exploring information visualization: describing different interaction patterns. In Proc. BELIV, pp. 16-23, 2010.

[96] Z. Pousman, J. Stasko, and M. Mateas. Casual information visualization: Depictions of data in everyday life. TVCG, 13(6):1145-1152, 2007.

[97] L. Ren, J. Cui, Y. Du, and G. Dai. Multilevel interaction model for hierarchical tasks in information visualization. In Proc. VINCI, pp. 11$16,2013$.
[98] R. E. Roth. Cartographic interaction primitives: Framework and synthesis. Cartogr. J., 49(4):376-395, 2012.

[99] R. E. Roth. An empirically-derived taxonomy of interaction primitives for interactive cartography and geovisualization. TVCG, 19(12):2356-2365, 2013.

[100] B. Saket, H. Kim, E. T. Brown, and A. Endert. Visualization by demonstration: An interaction paradigm for visual data exploration. TVCG, 23(1):331-340, 2017.

[101] B. Saket, A. Srinivasan, E. D. Ragan, and A. Endert. Evaluating interactive graphical encodings for data visualization. TVCG, 24(3):1316-1330, 2018.

[102] A. Satyanarayan, K. Wongsuphasawat, and J. Heer. Declarative interaction design for data visualization. In Proc. UIST, pp. 669-678, 2014.

[103] H.-J. Schulz, T. Nocke, M. Heitzler, and H. Schumann. A design space of visualization tasks. TVCG, 19(12):2366-2375, 2013.

[104] K. Sedig and P. Parsons. Interaction design for complex cognitive activities with visual representations: a pattern-based approach. Trans. Hum.-Comput. Interact., 5(2):84-133, 2013.

[105] K. Sedig, P. Parsons, M. Dittmer, and R. Haworth. Human-Centered Interactivity of Visualization Tools: Micro- and Macro-level Considerations. Springer, 2014.

[106] B. Shneiderman. The eyes have it: a task by data type taxonomy for information visualizations. In Proc. VL, pp. 336-343, 1996.

[107] B. Shneiderman. Promoting universal usability with multi-layer interface design. Comput. Phys. Handicap., (73-74):1-8, 2002.

[108] H. A. Simon. Models of man. Wiley, 1957.

[109] M. Spindler, C. Tominski, H. Schumann, and R. Dachselt. Tangible views for information visualization. In Proc. ITS, pp. 157-166, 2010.

[110] G. Teubner. Rights of non-humans? Electronic agents and animals as new actors in politics and law. J. Law Soc., 33(4):497-521, 2006.

[111] A. Thudt, D. Baur, S. Huron, and S. Carpendale. Visual mementos: Reflecting memories with personal data. TVCG, 22(1):369-378, 2016.

[112] C. Tominski. Interaction for visualization. Morgan \& Claypool, 2015.

[113] J. Tost and F. Heidmann. Visualization and interaction with multiple devices. A case study on reachability of remote areas for emergency management. $i$-com, 16(3):223-246, 2017.

[114] E. R. Tufte. The visual display of quantitative information. Graphics Press, 1983.

[115] J. van Wijk. Views on Visualization. TVCG, 12(4):421-432, 2006.

[116] F. B. Viegas, M. Wattenberg, F. van Ham, J. Kriss, and M. McKeon. ManyEyes: a site for visualization at internet scale. TVCG, 13(6):11211128, 2007.

[117] J. Walny, S. Huron, C. Perin, T. Wun, R. Pusch, and S. Carpendale. Active reading of visualizations. TVCG, 24(1):770-780, 2018.

[118] X. Wang, D. H. Jeong, W. Dou, S.-w. Lee, W. Ribarsky, and R. Chang. Defining and applying knowledge conversion processes to a visual analytics system. Computers \& Graphics, 33(5):616-623, 2009.

[119] Y. Wang, H. Zhang, H. Huang, X. Chen, Q. Yin, Z. Hou, D. Zhang, Q. Luo, and H. Qu. InfoNice: Easy creation of information graphics. In Proc. CHI, pp. 335:1-335:12, 2018.

[120] M. Ward and J. Yang. Interaction spaces in data and information visualization. In Proc. Eurographics, vol. 22, pp. 37-145, 2004.

[121] W. Willett, Y. Jansen, and P. Dragicevic. Embedded data representations. TVCG, 23(1):461-470, 2017.

[122] C. Williamson and B. Shneiderman. The dynamic HomeFinder. In Proc. SIGIR, pp. 338-346, 1992.

[123] M. Wybrow, N. Elmqvist, J.-D. Fekete, T. von Landesberger, J. J. van Wijk, and B. Zimmer. Interaction in the visualization of multivariate networks. In LNCS, vol. 8380, pp. 97-125. 2014.

[124] H. Xia, N. Henry Riche, F. Chevalier, B. De Araujo, and D. Wigdor. Dataink: Direct and creative data-oriented drawing. In Proc. CHI, pp. 223:1-223:13, 2018.

[125] A. Yelizarov and D. Gamayunov. Adaptive visualization interface that manages user's cognitive load based on interaction characteristics. In Proc. VINCI, pp. 1:1-1:8, 2014.

[126] J. S. Yi, Y. ah Kang, and J. Stasko. Toward a deeper understanding of the role of interaction in information visualization. TVCG, 13(6):1224-1231, 2007.

[127] J. S. Yi, R. Melton, J. Stasko, and J. A. Jacko. Dust\&magnet: Multivariate information visualization using a magnet metaphor. Inf. Vis., 4(4):239256, 2005. 